\title{
Reasons That Life is Difficult
}

\author{
Kathryn D. Winters
}

Published online: 13 October 2011

(C) Springer Science+Business Media, LLC 2011

1. Not enough time to wade through undergraduate medical education with care.

2. Lab partner talks loudly and wants our group to do everything very quickly.

3. I don't like the guy who likes me.

4. Nose gets red in the cold, in the hot, or if I am really tired and/or have to pee.

5. Eyes require glasses.

6. Bayes' Theorem is confusing.

7. Terrible tooth pain upon consumption of sugar.

8. Near constant consumption of sugar.

9. Locked myself out of the house twice in the span of 7 days.

10. When called on in small group, could not remember boundaries of eye's anterior chamber.

11. Family relationships.

12. Cat bit my face.

13. Rapidly declining reproductive fitness. Also-bone mass tapped out.

14. Charles Bukowski is dead.

K. D. Winters

UCSD School of Medicine, University of California, La Jolla, CA, USA

e-mail: kdwinters@ucsd.edu 